\title{
Interval Type-2 Fuzzy Model Based on Inverse Controller Design for the Outlet Temperature Control System of Ethylene Cracking Furnace
}

\author{
Taoyan Zhao ${ }^{1, *}$, Ping $\mathrm{Li}^{2, *}$, Jiangtao $\mathrm{Cao}^{2}$ and Tian $\mathrm{Li}^{3}$ \\ 1 School of Automation, Northwestern Polytechnical University, Xi'an 710129, China \\ 2 School of Information and Control Engineering, Liaoning Shihua University, Fushun 113001, China; \\ jtcao@lnpu.edu.cn \\ 3 Sinopec Tianjin Natural Gas Pipe Company, Tianjin 300457, China; cigroup@126.com \\ * Correspondence: zhaotaoyan1986@126.com (T.Z.); liping@lnpu.edu.cn (P.L.)
}

Received: 30 August 2017; Accepted: 20 September 2017; Published: 22 September 2017

\begin{abstract}
Multivariable coupling, nonlinear and large time delays exist in the coil outlet temperature (COT) control system of the ethylene cracking furnace, which make it hard to achieve accurate control over the COT of the furnace in actual production. To solve these problems, an inverse controller based on an interval type-2 fuzzy model control strategy is introduced. In this paper, the proposed control scheme is divided into two parts: one is the approach structure part of the interval type-2 fuzzy model (IT2-FM), which is utilized to approach the process output. The other is the interval type-2 fuzzy model inverse controller (IT2-FMIC) part, which is utilized to control the output process to achieve the target value. In addition, on the cyber-physical system platform, the actual industrial data are used to test and obtain the mathematical model of the COT control system of the ethylene cracking furnace. Finally, the proposed inverse controller based on the IT2-FM control scheme has been implemented on the COT control system of the ethylene cracking furnace, and the simulation results show that the proposed method is feasible.
\end{abstract}

Keywords: interval type-2 fuzzy model; inverse controller; cyber-physical system; ethylene cracking furnace; COT control system

\section{Introduction}

As an important and common raw material in the petrochemical industry, ethylene has a peculiar position in the development of the economy and society. The cracking furnace is the key unit of an ethylene plant, and its control performance directly affects the ethylene yield and operational stabilization of the related following work section. The ethylene cracking furnace mainly consists of a convection segment and a radiation segment. The raw oil is preheated and vaporized in the convection segment, then mixed with dilution steam into the radiation segment. The radiation segment is the reaction area and divided into eight zones; each zone can be heated independently to track the target temperature curve. The schematic diagram of the ethylene cracking furnace is shown in Figure 1. The cracking depth value is the most important control objective for an ethylene cracking furnace. However, it is difficult to obtain the accurate the cracking depth value online in the actual production process. So far, the average of the coil outlet temperature (COT) is usually used as the index to control the running of the ethylene cracking furnace [1]. However, the cracking process is a complex of chemical changes with many issues that are hard to solve by a conventional control scheme, such as the large deviation of the furnace tube outlet temperature, nonlinear performance with the process of the coking furnace tube and the multi-input and multi-output behavior [2]. Thus, it is necessary 
to adopt an advanced process control scheme to solve these challenges, which is also the inevitable course of the current petrochemical industry.

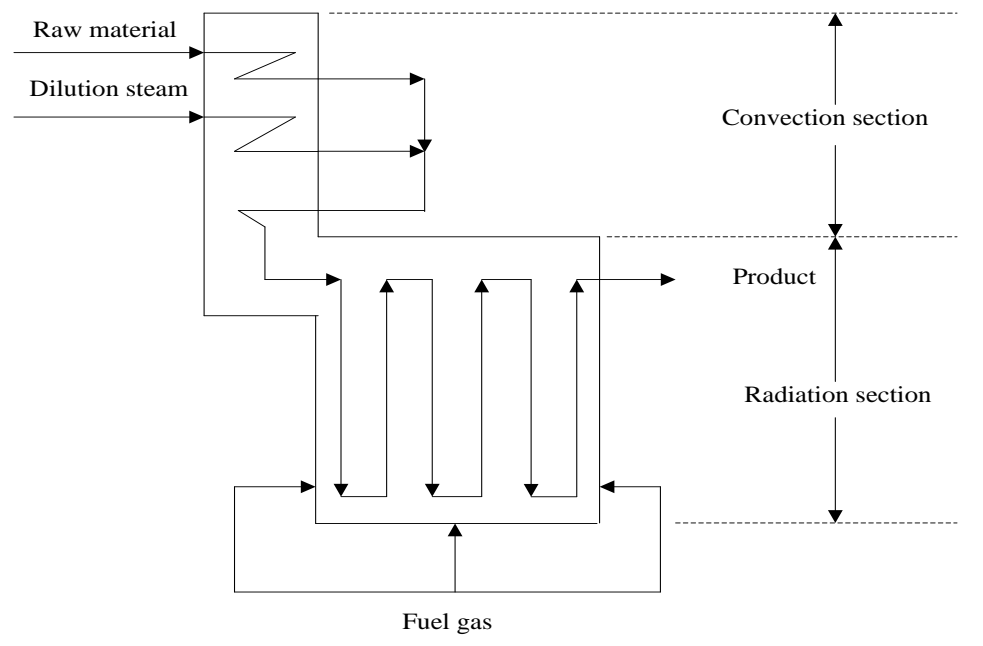

Figure 1. Structure diagram of the ethylene cracking furnace.

Recently, the interval type-2 fuzzy logic system (IT2-FLS) has been widely applied in a variety of fields, such as the $\mathrm{pH}$ neutralization process [3,4], the nonlinear continuous bioreactor with bifurcation [5], the mobile robot [6,7], the coupled-tank system [8], the temperature control [9], the double-inverted pendulum [10], aerospace [11], the classification and curve identification datasets [12], airplane flight control [13], the travelling salesman problem [14], power management and electrical control [15], benchmark control problems [16], and so on. The IT2-FLS extends the reasoning and design freedom of the fuzzy system because of the expanding dimension performance provided by the footprint of uncertainty (FOU) [17]. In [18-20], it has been shown that IT2-FLS is superior for handling uncertainties and nonlinearities compared with the conventional fuzzy logic system.

On the other hand, the high computational cost of the type-reduction process of an IT2-FLS is a major bottleneck [21], which may hinder its application in the real world. At present, scholars mainly utilize the K-M iterative type-reduction algorithm [22] and the $\mathrm{Wu}-\mathrm{Mendel}$ uncertain boundary type-reduction algorithm [23] in the interval type-2 fuzzy controllers. The former can offer an exact computation of the type-reduction sets, but the high computational cost of the iterative computational process in type-reduction means that it will limit the application of the IT2-FLS, especially for the high real-time requirements of the system; the latter is an alternative type-reduction algorithm, which has closed-form expressions by calculating the inner-bound and outer-bound sets. Thus, the Wu-Mendel type-reduction algorithm is usually faster than the K-M type-reduction algorithm and has the potential to reduce the computational burden of the IT2-FLS. In this paper, the $\mathrm{Wu}-\mathrm{Mendel}$ type-reduction algorithm is adopted in the proposed controller.

Internal model control was put forward by Garcia and Morari in 1982 [24], which is one of the most commonly-used control structures in the process control field. Its main characteristic is the simple structure and design, excellent control performance and disturbance rejection capability, especially for a nonlinear large-delay system. Therefore, many scholars have had a strong interest in internal model control and also have achieved many research results [25-28]. However, there is the thorny problem of finding a process model that represents the actual nonlinear system in the internal model control structure. In general, scholars mostly adopt the mathematical model approach to the process model, but process objects of industry are generally uncertain, and it is sometimes difficult to build a mathematical model especially for the actual petrochemical process. In order to solve this problem, some scholars use the main controllers based on the fuzzy model in the internal model control structure, which have achieved good results in practical applications [29-31]. In [4,17,32,33], 
some inverse controllers based on a type-2 fuzzy model control design strategy are developed, which have been successfully applied to the $\mathrm{pH}$ neutralization process and the spherical tank process. In [34], a new iterative type-2 fuzzy model inversion technique based on the big bang big crunch (BB-CC) is introduced, which is feasible through the simulation study of the level control of a spherical tank.

In this article, a novel inverse controller design scheme based on IT2-fuzzy model (FM) has been developed. The proposed control scheme is divided into two parts: one is the approach structure part of IT2-FM, which is utilized to approach the complex nonlinear process output by exploiting the advantages of IT2-FM in dealing with nonlinear and uncertain problems. The other part is the interval type-2 fuzzy model inverse controller (IT2-FMIC) design, which is utilized to control the output process to achieve the target value by exploiting the advantages of IT2-FLS and the internal model structure. Finally, the proposed IT2-FM approach structure and IT2-FMIC are applied to the COT control system of the ethylene cracking furnace. The performance of the proposed approach structures of IT2-FM and IT2-FMIC are compared with the corresponding type- 1 method. The comparison results show that the proposed method can obtain better approach and control performance.

This paper is organized into six sections. In Section 2, the architecture of IT2-FM is introduced. The approach strategy of IT2-FM is presented in Section 3. Then, in Section 4, the newly proposed inverse controller based on IT2-FM is explained in detail. In Section 5, the proposed approach structures of IT2-FM and IT2-FMIC are implemented and tested on the outlet temperature of the ethylene cracking furnace. Finally, the conclusion is given.

\section{Interval Type-2 Fuzzy Set and Model}

The type-2 fuzzy set (T2-FS) was first introduced by Zadeh, which can be seen as an extension of the traditional fuzzy set $[35,36]$. Compared with the traditional fuzzy set, the membership function (MF) of T2-FS is one fuzzy set rather than a precise value. The MF of general T2-FS is composed of the main MF and the secondary MF. Interval type-2 fuzzy set (IT2-FS) as a special case is characterized as: the secondary MF degree of T2-FS is one, so its related computing and operation are simpler than the corresponding type-2 counterpart. An illustration of an IT2-FS is show in Figure 2. Observe that the IT2-FS is bounded from above and below by two type-1 fuzzy sets, which are called the upper MF (UMF) and the lower MF (LMF), respectively. The footprint of uncertainty (FOU) consists of the area between $\operatorname{UMF}(\widetilde{A})$ and $\operatorname{LMF}(\widetilde{A})$.

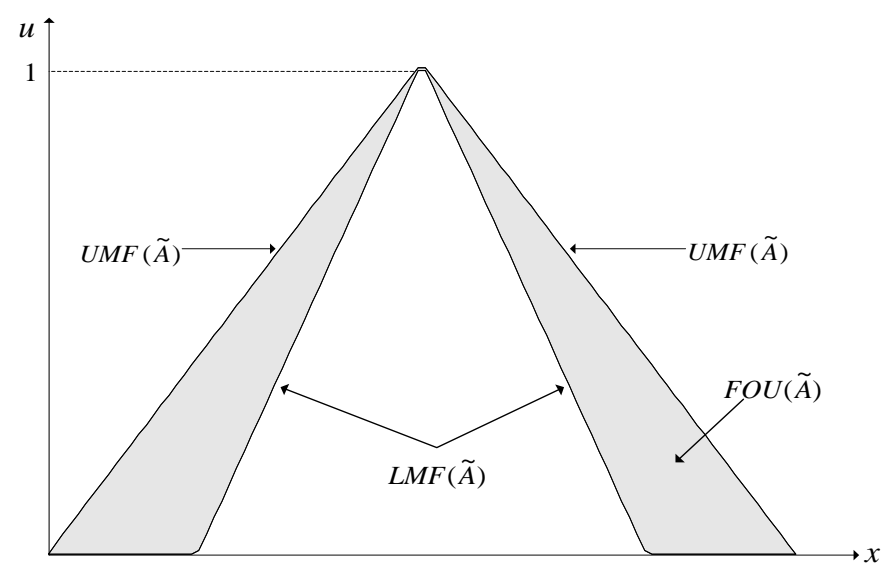

Figure 2. Illustration of interval type-2 (IT2)-membership function (MF). UMF, upper MF; LMF, lower MF; FOU, footprint of uncertainty.

The general structure of IT2-FM is shown in Figure 3. Unlike the conventional fuzzy system, the output of the IT2-FM is an IT2-FS, which needs to be made into the type- 1 counterpart by the type-reduction process. 


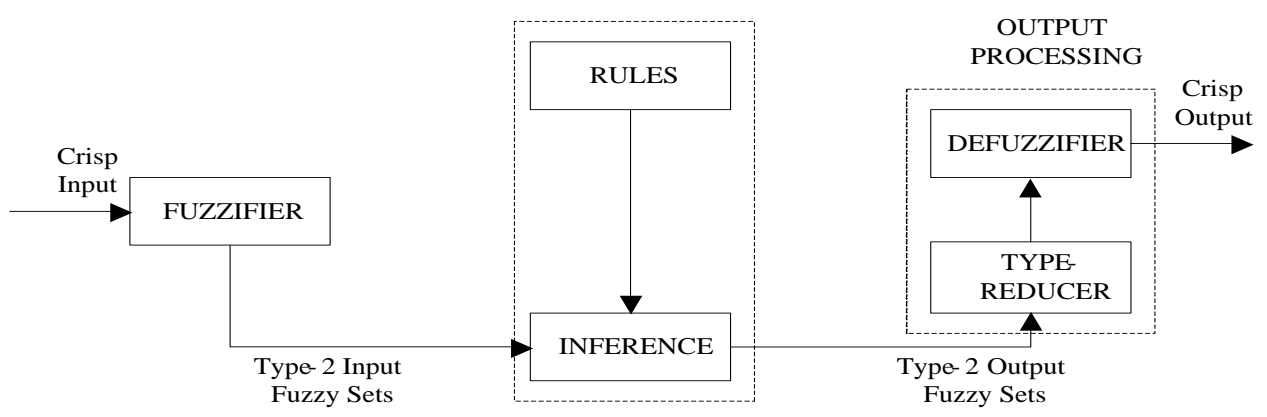

Figure 3. Structure diagram of the IT2-fuzzy model (FM).

An IT2-FM rule can be expressed as follows:

$$
r_{j}: \text { IF } \mathrm{x}_{1} \text { is } \widetilde{A}_{1} \text { and } x_{2} \text { is } \widetilde{A}_{2} \text { and } \cdots x_{n} \text { is } \widetilde{A}_{n} \text { THEN } y \text { is } C^{j}, j=1,2 \cdots, N
$$

where $\widetilde{A}_{1}, \widetilde{A}_{2}, \ldots, \widetilde{A}_{n}$ are antecedent MFs of the IT2-FM, while $C^{j}$ is the consequent MF and usually used with centroid representation, and $N$ is the total number of fuzzy rules.

$$
\begin{gathered}
\widetilde{\mu}_{1}=\left[\mu \widetilde{A}_{1}, \bar{\mu} \widetilde{A}_{1}\right] \\
\widetilde{\mu}_{2}=\left[\underline{\mu} \widetilde{A}_{2}, \bar{\mu} \widetilde{A}_{2}\right] \\
\vdots \\
\widetilde{\mu}_{n}=\left[\mu \widetilde{A}_{n}, \bar{\mu} \widetilde{A}_{n}\right]
\end{gathered}
$$

where $\underline{\mu}_{j}$ and $\bar{\mu}_{j}$ are the lower and upper firing intervals, respectively. The total of the firing intervals of IT2-FM is described as:

$$
\begin{gathered}
f^{j}=\left[\underline{f}^{j} \bar{f}^{j}\right] \\
\bar{f}^{j}=\underline{\mu}_{1}\left(x_{1}\right) \cap \underline{\mu}_{2}\left(x_{2}\right) \cap \underline{\mu}_{n}\left(x_{n}\right) \\
\bar{f}^{j}=\bar{\mu}_{1}\left(x_{1}\right) \cap \bar{\mu}_{2}\left(x_{2}\right) \cap \bar{\mu}_{n}\left(x_{n}\right)
\end{gathered}
$$

The consequent $C^{j}$ of the rule of the IT2-FM is the interval set, defined as:

$$
C^{j}=\left[c_{l}^{j}, c_{r}^{j}\right]
$$

The center-of-sets is one of the most popular methods for an IT2-FM. Thus, the type-reduced set for the IT2-FM is expressed as:

$$
Y_{T R}=\left[y_{l}(x), y_{r}(x)\right]
$$

where $Y_{T R}$ is an interval type-reduced set, and it is determined by its two end points $y_{l}(x)$ and $y_{r}(x)$. The end-points $y_{l}(x)$ and $y_{r}(x)$ of the $Y_{T R}$ of an IT2-FM for the input $x$ are bounded from below and above by $\underline{y}_{l}(x) \leq y_{l}(x) \leq \bar{y}_{l}(x)$ and $\bar{y}_{r}(x) \leq y_{r}(x) \leq \underline{y}_{r}(x)$. The inner-bound sets of $Y_{T R}$ are represented as:

$$
\begin{aligned}
& \bar{y}_{l}(x)=\min \left\{y_{l}^{(M)}(x), y_{l}^{(0)}(x)\right\} \\
& \underline{y}_{r}(x)=\max \left\{y_{r}^{(M)}(x), y_{r}^{(0)}(x)\right\}
\end{aligned}
$$

where:

$$
\begin{aligned}
& y_{l}^{(0)}(x)=\sum_{i=1}^{M} \underline{f}^{i} y_{l}^{i} / \sum_{i=1}^{M} \underline{f}^{i} \\
& y_{l}^{(M)}(x)=\sum_{i=1}^{M} \bar{f}^{i} y_{l}^{i} / \sum_{i=1}^{M} \bar{f}^{i}
\end{aligned}
$$




$$
\begin{aligned}
& y_{r}^{(0)}(x)=\sum_{i=1}^{M} \underline{f}^{i} y_{r}^{i} / \sum_{i=1}^{M} \underline{f}^{i} \\
& y_{r}^{(M)}(x)=\sum_{i=1}^{M} \bar{f}^{i} y_{r}^{i} / \sum_{i=1}^{M} \bar{f}^{i}
\end{aligned}
$$

The outer-bound sets of $Y_{T R}$ are represented as:

$$
\begin{aligned}
& \underline{y}_{l}(x)=\bar{y}_{l}(x)-\left[\begin{array}{l}
\sum_{i=1}^{M}\left(\bar{f}^{i}-\underline{f}^{i}\right) \\
\sum_{i=1}^{M} \bar{f}^{i} \sum_{i=1}^{M} \underline{f}^{i}
\end{array} \times \frac{\sum_{i=1}^{M} \underline{f}^{i}\left(y_{l}^{i}-y_{l}^{1}\right) \sum_{i=1}^{M} \bar{f}^{i}\left(y_{l}^{M}-y_{l}^{i}\right)}{\sum_{i=1}^{M} \underline{f}^{i}\left(y_{l}^{i}-y_{l}^{1}\right)+\sum_{i=1}^{M} \bar{f}^{i}\left(y_{l}^{M}-y_{l}^{i}\right)}\right] \\
& \bar{y}_{r}(x)=\underline{y}_{r}(x)+\left[\frac{\sum_{i=1}^{M}\left(\bar{f}^{i}-\underline{f}^{i}\right)}{\sum_{i=1}^{M} \bar{f}^{i} \sum_{i=1}^{M} \underline{f}^{i}} \times \frac{\sum_{i=1}^{M} \bar{f}^{i}\left(y_{r}^{i}-y_{r}^{1}\right) \sum_{i=1}^{M} \underline{f}^{i}\left(y_{r}^{M}-y_{r}^{i}\right)}{\sum_{i=1}^{M} \bar{f}^{i}\left(y_{r}^{i}-y_{r}^{1}\right)+\sum_{i=1}^{M} \underline{f}^{i}\left(y_{r}^{M}-y_{r}^{i}\right)}\right]
\end{aligned}
$$

The approximation of the type-reduced set is shown as follows:

$$
\left[y_{l}(x), y_{r}(x)\right] \approx\left[\frac{\bar{y}_{r}(x)+\underline{y}_{r}(x)}{2}, \frac{\underline{y}_{l}(x)+\bar{y}_{l}(x)}{2}\right]
$$

The precise output of the IT2-FM is defined as:

$$
y(x)=\frac{1}{2}\left[\frac{\bar{y}_{r}(x)+\underline{y}_{r}(x)}{2}+\frac{\underline{y}_{l}(x)+\bar{y}_{l}(x)}{2}\right]
$$

\section{Approach Structure of the Interval Type-2 Fuzzy Model}

The choice of process model inversion is crucial for the internal model control structure. However, the choice of process model inversion is difficult for some complex uncertain systems. IT2-FM is a powerful tool that deals with highly nonlinear and uncertain processes. In this paper, the IT2-FM is utilized to approach the complex process output. The structure diagram of the approach strategy of IT2-FM is shown in Figure 4.

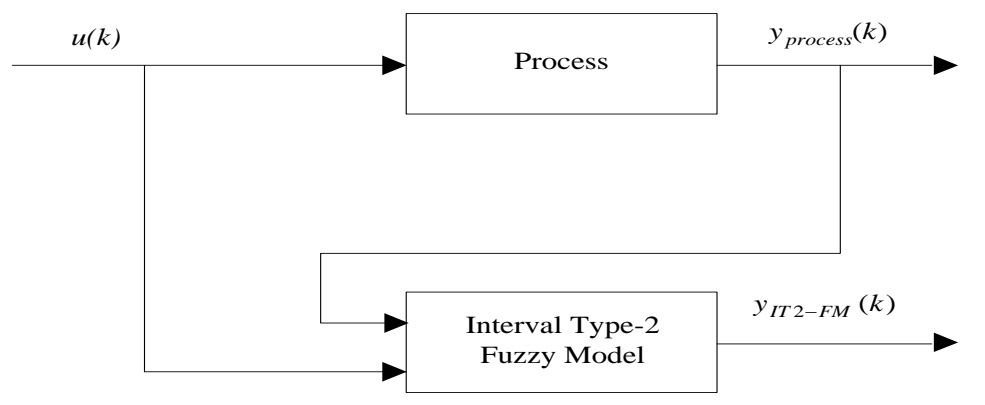

Figure 4. Open loop approach structure diagram of IT2-FM.

Let us design a simple IT2-FM with two inputs $\left(u(k), y_{P R O C E S S}(k)\right)$ and one output $\left(y_{I T 2-F M}(k)\right)$. The $u(k)$ and $y_{P R O C E S S}(k)$ represent the output of the inverse controller to be designed and process output, respectively. The $y_{I T 2-F M}(k)$ represents the output of the IT2-FM, which is utilized to approach the output of the original process. The antecedent MFs for the inputs $u(k)$ and $y_{P R O C E S S}(k)$ are shown in Figure 5. The total number of the fuzzy rules is $3 \times 4=12$. The rule base and consequents are given in Table 1. The reasoning process of the IT2-FM is described in detail in Section 2. 

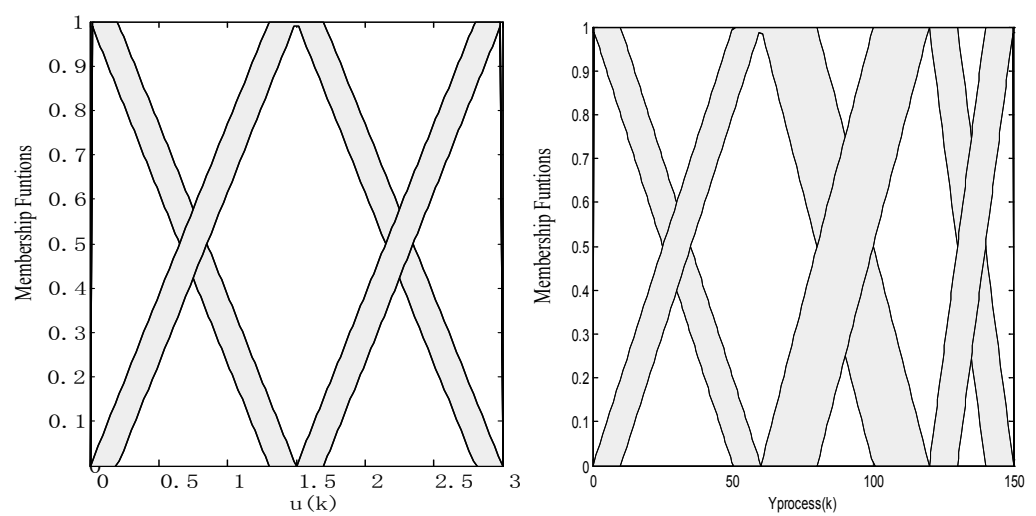

Figure 5. Antecedent membership functions of the IT2-FM.

Table 1. The rule base and consequents of the IT2-FM for an illustrative example.

\begin{tabular}{cccc}
\hline \multirow{2}{*}{$(\boldsymbol{k})$} & \multicolumn{3}{c}{$\boldsymbol{u}(\boldsymbol{k})$} \\
\cline { 2 - 4 } & $\widetilde{\boldsymbol{B}}_{\mathbf{1}}^{\mathbf{1}}$ & $\widetilde{\boldsymbol{B}}_{\mathbf{1}}^{\mathbf{2}}$ & $\widetilde{\boldsymbol{B}}_{\mathbf{1}}^{\mathbf{3}}$ \\
\hline$\widetilde{A}_{1}^{1}$ & $c^{1}=[0,30]$ & $c^{2}=[20,40]$ & $c^{3}=[40,120]$ \\
$\widetilde{A}_{1}^{2}$ & $c^{4}=[120,130]$ & $c^{5}=[0,30]$ & $c^{6}=[30,70]$ \\
$\widetilde{A}_{1}^{3}$ & $c^{7}=[60,120]$ & $c^{8}=[110,150]$ & $c^{9}=[5,30]$ \\
$\widetilde{A}_{1}^{4}$ & $c^{10}=[40,120]$ & $c^{11}=[123,132]$ & $c^{12}=[130,150]$ \\
\hline
\end{tabular}

In order to test the approach structure of IT2-FM, it is examined via simulations on a mathematical model. Many complex processes, especially petrochemical processes, can be described by a first order plus large lag model, as shown in the following:

$$
G(s)=\frac{37}{29 s+1} e^{-35 s}
$$

Then, the step signal is adopted in order to activate IT2-FM. The approach result is illustrated in Figure 6.

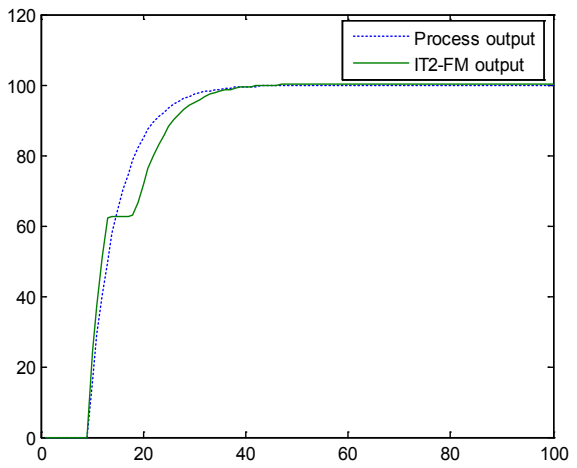

Figure 6. IT2-FM output approach process output.

From Figure 6, it can be seen that the approach results of the IT2-FM reach a certain requirement, so the IT2-FM approach scheme is effective.

\section{Inverse Interval Type-2 Fuzzy Controller Design}

The simplest way to design a model-based controller for a process is to set up its inverse model as the controller [37]. Let us design a simple IT2-FM, which is expressed as follows: 


$$
y(k)=f(x(k), u(k))
$$

where $x(k)$ is the state vector, $u(k)$ is the current input, $y(k)$ is the output and $f$ denotes the fuzzy mapping. Here, the inverse controller is formed utilizing the IT2-FM, which is shown in Figure 7.

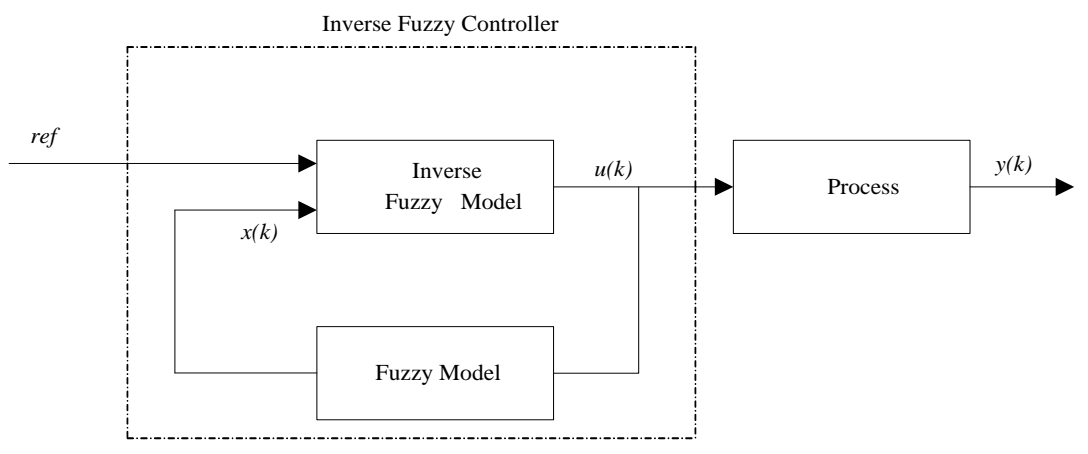

Figure 7. Open loop structure of the IT2-FM inverse controller.

Since the IT2-FM has a stronger ability to deal with a dynamic process compared with T1-FM, the control performance of the inverse controller based on IT2-FM is superior to the corresponding type-1 counterpart. In Section 3, it has been verified that the approach structure of the IT2-FM is valid, i.e., $y(k)=r e f$. Thus, if the IT2-FM approaches the process exactly, the process output would converge to the reference value. The structure of proposed inverse controller based on IT2-FM is shown in Figure 8, which provides an effective control performance $[4,17,37]$.

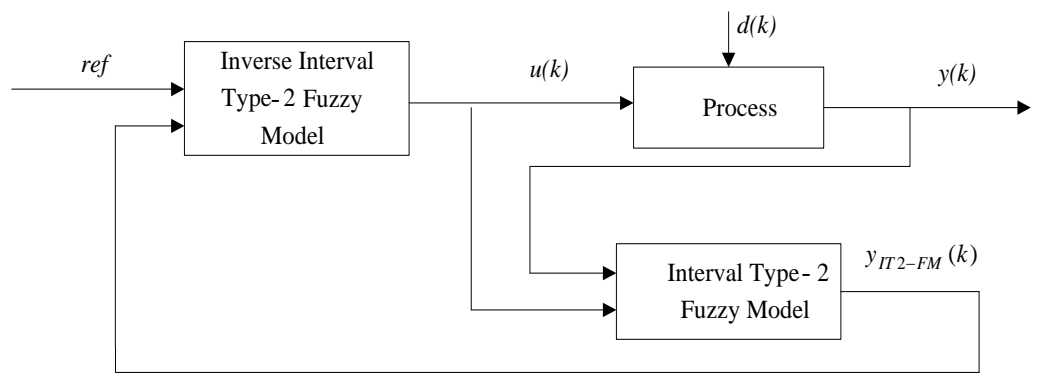

Figure 8. Inverse controller based on the IT2-FM design scheme.

\section{Simulation Studies}

A cyber-physical system (CPS) is a new complex system with heterogeneous computing units and physical objects, which are highly integrated and interactive in the network environment [38]. In the control of the ethylene cracking furnace outlet temperature system, the construction of communication, computing and control between the physical layer and the information layer is an essential part. The advanced process control system is implemented in an industrial upper computer, which is connected with the object linking and embedding for process control (OPC) server through the hub. The physical link of data transmission is established between the OPC server and the control station through the internal protocol; thus, advanced process control system upper computer utilizes the corresponding communication configuration, which can achieve the physical link of data transmission with the OPC server. The data exchange between the advanced process control system and the DCS control station is realized by the OPC standard interface. The block diagram of the CPS is shown in Figure 9. A large amount of field data is collected and saved in the upper computer via CPS. 
The mathematical model of the COT control system of the ethylene cracking furnace is obtained by using the step signal as the test signal:

$$
G(s)=\frac{5.2634}{146.97 s+1} e^{-6.7259 s}
$$

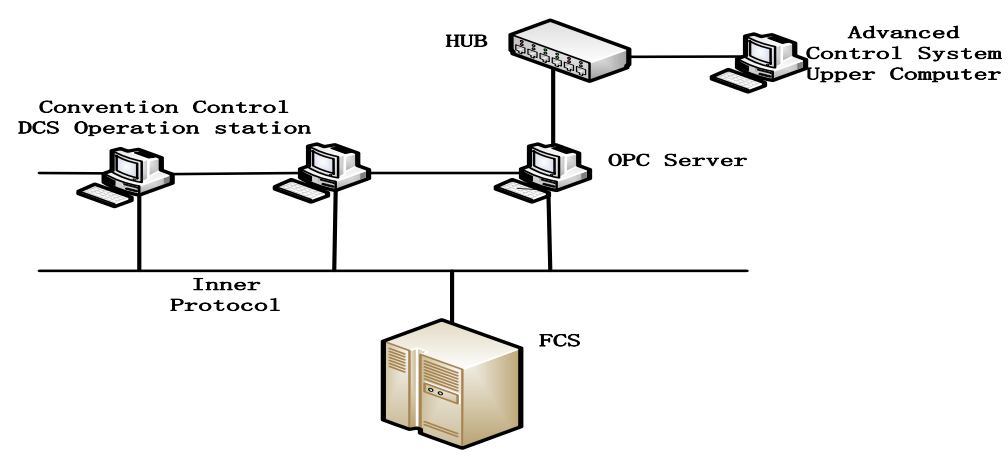

Figure 9. The block diagram of the cyber-physical system (CPS).

\subsection{Performance Comparison of Type-1 and Interval Type-2 Fuzzy Approach Models}

In this part, the effectiveness of the interval type-2 fuzzy approach model for the COT control system of the ethylene cracking furnace will be demonstrated. The approach structure of the fuzzy model is a system with two inputs (inverse controller output, process output) and one output (fuzzy model output), as shown in Figure 4. The antecedent MFs for the inputs are shown in Figure 10. The consequent part of the fuzzy rule is illustrated with 12 interval sets, which are illustrated in Table 2. The total number of rules is 12 . The approach process is similar to the third part of the paper. The process output and fuzzy model outputs are added to the disturbances, respectively. The approach results are illustrated in Figure 11.
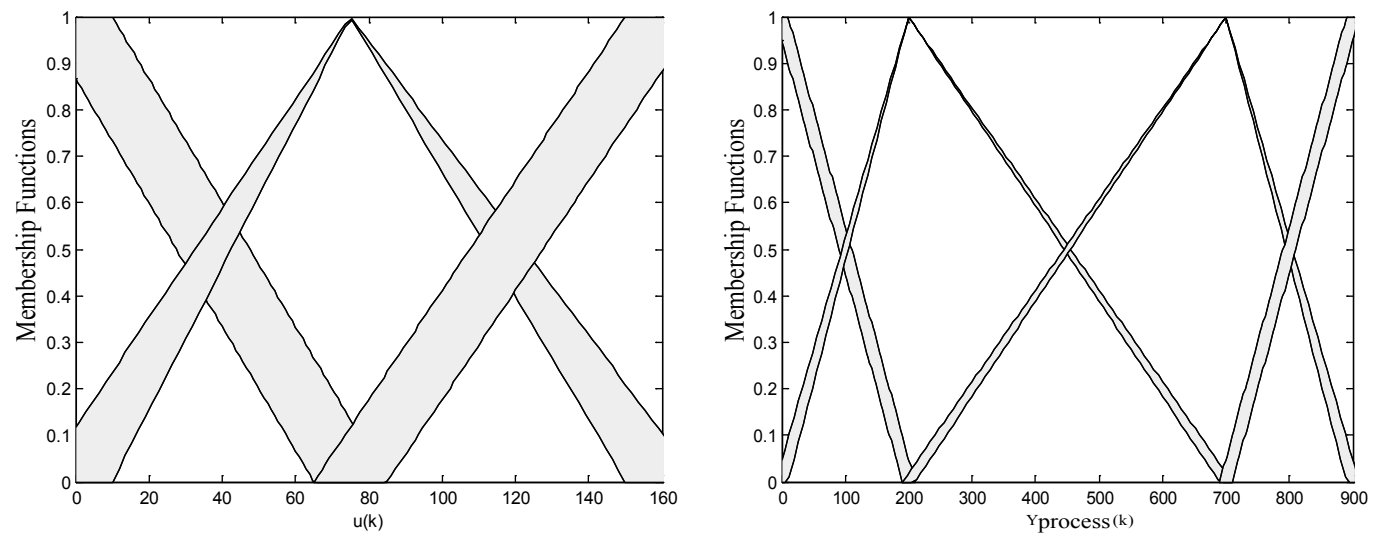

Figure 10. Antecedent membership functions of the IT2-FM.

Table 2. The rule base and consequents of the IT2-FM.

\begin{tabular}{cccc}
\hline & & \multicolumn{3}{c}{$\boldsymbol{u}(\boldsymbol{k})$} & \\
\cline { 2 - 4 } & $\widetilde{\boldsymbol{B}}_{\mathbf{1}}^{\mathbf{1}}$ & $\widetilde{\boldsymbol{B}}_{\mathbf{1}}^{\mathbf{2}}$ & $\widetilde{\boldsymbol{B}}_{\mathbf{1}}^{\mathbf{3}}$ \\
\hline$\widetilde{A}_{1}^{1}$ & $c^{1}=[22.636,32.915]$ & $c^{5}=[22.636,32.915]$ & $c^{9}=[22.636,32.915]$ \\
$\widetilde{A}_{1}^{2}$ & $c^{2}=[226.66,240.02]$ & $c^{6}=[226.66,240.02]$ & $c^{10}=[226.66,240.02]$ \\
$\widetilde{A}_{1}^{3}$ & $c^{3}=[543.33,556.66]$ & $c^{7}=[543.33,556.66]$ & $c^{11}=[543.33,556.66]$ \\
$\widetilde{A}_{1}^{4}$ & $c^{4}=[800.00,812.59]$ & $c^{8}=[800.00,812.59]$ & $c^{12}=[800.00,812.59]$ \\
\hline
\end{tabular}




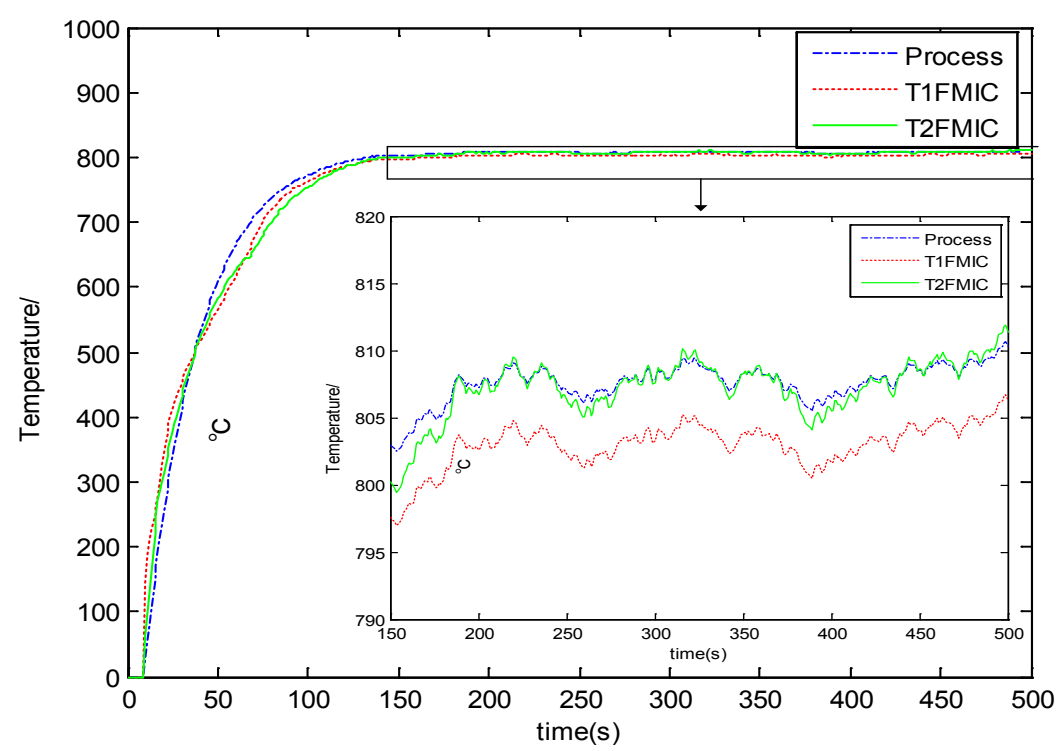

Figure 11. The output comparison of the process, type-1 fuzzy model (T1-FM) and IT2-FM.

In order to make a fair comparison between T1-FM and IT2-FM, two performance indexes are given.

(1) Root mean square error (RMSE), which is described as the following formula:

$$
R M S E=\sqrt{\frac{1}{n} \sum_{k=1}^{n}\left(y_{p}-y_{F M}\right)^{2}}
$$

(2) Variance accounted for (VAF) [4], which is described as the following formula:

$$
V A F=100 \% \times\left[1-\frac{\operatorname{var}\left(y_{p}-y_{F M}\right)}{\operatorname{var}\left(y_{p}\right)}\right]
$$

where $y_{p}$ represents the process output and $y_{F M}$ represents the fuzzy model output. The VAF index is usually utilized to evaluate the performance of a model, by comparing the real process output with the model output. If the two signals are closer, then the VAF value is bigger.

The performance results obtained from Formulas (20) and (21) are shown in Table 3. It can be clearly seen that the IT2-FM has a smaller RMSE value and a higher VAF value compared to the corresponding T1-FM. Therefore, it is seen obviously that IT2-FM has better approximation accuracy than T1-FM. Here, it is illustrated in Figure 11 that the IT2-FM represents the outlet temperature in the ethylene cracking furnace obviously better than the T1-FM.

Table 3. Comparison of the fuzzy models. VAF, variance accounted for.

\begin{tabular}{ccc}
\hline- & RMSE & VAF \\
\hline T1-FM & 22.0541 & 0.9816 \\
IT2-FM & 16.2918 & 0.9900 \\
\hline
\end{tabular}

5.2. Control Performance Evaluation of the T1-FMIC and IT2-FMIC Strategies for the Outlet Temperature of the Ethylene Cracking Furnace

It is a known fact that the ethylene cracking furnace is a complex industrial process with nonlinearity and time delay. The control goal for the coking furnace is to stabilize the outlet 
temperature, to improve its disturbance rejection capability and to prolong the run time of the furnace. Here, the proposed IT2-FMIC strategy is applied to the COT control system of the ethylene cracking furnace. The proposed IT2-FMIC has two inputs (set value of COT, fuzzy model output) and one output (control variable); the antecedent MFs for the inputs are shown in Figure 12. The consequent part of the fuzzy rule is illustrated with 16 interval sets, which are illustrated in Table 4 . The total number of rules is 16 . The performance of the fuzzy inverse model controller is illustrated in Figure 13.

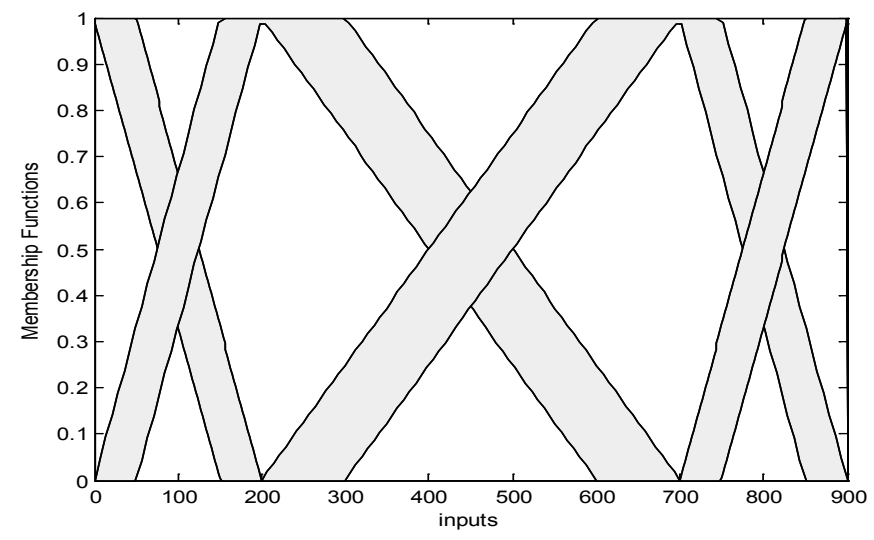

Figure 12. Antecedent membership functions of the inputs.

Table 4. The rule base and consequents of the proposed IT2-FMIC.

\begin{tabular}{ccccc}
\hline & \multicolumn{4}{c}{$\boldsymbol{u}(\boldsymbol{k})$} \\
\cline { 2 - 5 }$y(\boldsymbol{k})$ & $\widetilde{\boldsymbol{B}}_{\mathbf{1}}^{\mathbf{2}}$ & $\widetilde{\boldsymbol{B}}_{\mathbf{1}}^{\mathbf{3}}$ & $\widetilde{\boldsymbol{B}}_{\mathbf{1}}^{\mathbf{4}}$ \\
\hline$\widetilde{A}_{1}^{1}$ & $c^{1}=[14.44,24.59]$ & $c^{2}=[91.84,105.4]$ & $c^{3}=[128.8,143.3]$ & $c^{4}=[163.4,174.0]$ \\
$\widetilde{A}_{1}^{2}$ & $c^{5}=[14.44,24.59]$ & $c^{6}=[91.84,105.4]$ & $c^{7}=[91.84,105.4]$ & $c^{8}=[128.8,143.3]$ \\
$\widetilde{A}_{1}^{3}$ & $c^{9}=[91.84,105.4]$ & $c^{10}=[128.8,143.3]$ & $c^{11}=[163.4,174.0]$ & $c^{12}=[163.4,174.0]$ \\
$\widetilde{A}_{1}^{4}$ & $c^{13}=[91.84,105.4]$ & $c^{14}=[128.8,143.3]$ & $c^{15}=[163.4,174.0]$ & $c^{16}=[163.4,174.0]$ \\
\hline
\end{tabular}

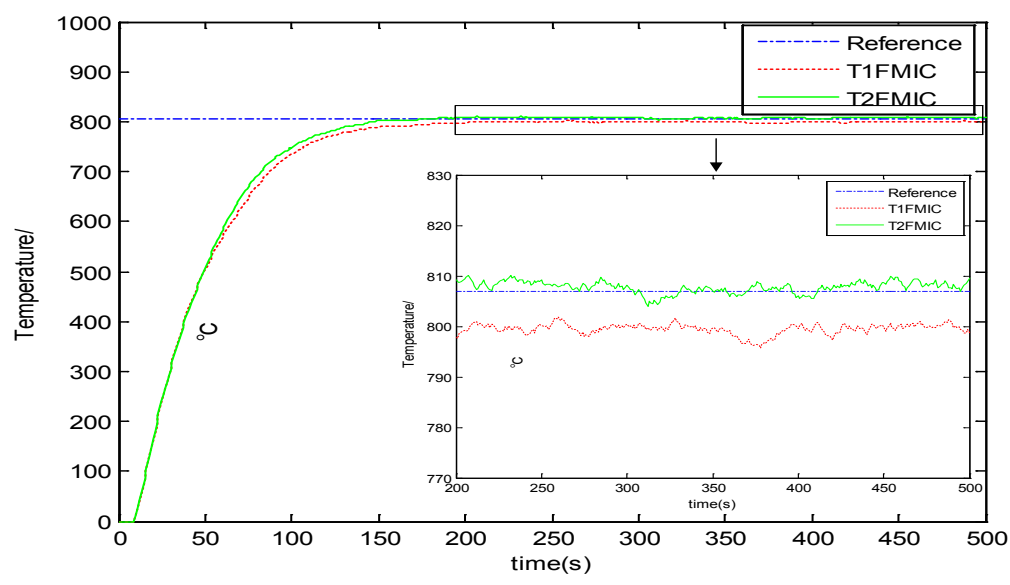

Figure 13. Tracking curve of IT1-FMIC and IT2-FMIC.

In order to make a fair comparison between the T1-FMIC scheme and the IT2-FMIC scheme, the performance indexes are considered as follows: 
(1) Integral of the square error (ISE), which is described as:

$$
I S E=\int_{0}^{\infty}\left((r(t)-y(t))^{2}\right) d t
$$

(2) Integral of the absolute error (IAE), which is described as:

$$
I A E=\int_{0}^{\infty}|r(t)-y(t)| d t
$$

(3) Integral of the time-weighted absolute error (ITAE), which is defined as:

$$
\text { ITAE }=\int_{0}^{\infty} t|r(t)-y(t)| d t
$$

where $r(t)$ and $y(t)$ are the target profile and the process output, respectively.

It has been illustrated in Figure 13 that the IT2-FMIC strategy has better control performances compared with the T1-FMIC strategy. Moreover, the performance index value of the IT2-FMIC is superior to the corresponding value of T1-FMIC, as seen in Table 5.

Table 5. Regulation performance comparison of the inverse controllers. ISE, integral of the square error; IAE, integral of the absolute error; ITAE, integral of the time-weighted absolute error.

\begin{tabular}{cccc}
\hline - & ISE & IAE & ITAE \\
\hline T1-MIC & $1.3544 \times 10^{7}$ & $3.3798 \times 10^{4}$ & $2.1988 \times 10^{6}$ \\
IT2-MIC & $1.3241 \times 10^{7}$ & $3.0134 \times 10^{4}$ & $1.3272 \times 10^{6}$ \\
\hline
\end{tabular}

\section{Conclusions}

This article is focused on IT2-FMIC design and its application in the outlet temperature control system of the ethylene cracking furnace, which is a typical nonlinear object in petrochemical industry. The proposed IT2-FMIC scheme is divided into two parts: the approach part and the control part. Simulation results reveal that the IT2-FM can accurately approximate the complex nonlinear chemical process, and the proposed IT2-FMIC scheme has better disturbance rejection capability. However, the proposed control strategy is still constrained to a single output process. In future work, extension of the proposed method to a multi-input multi-output process will be a hot issue. Then, the optimization of the inverse IT2-FM and its robustness analysis will also be the focus of research.

Acknowledgments: This work is supported by the National Science Foundation of China under Grant No. 61673199 and the Program for Liaoning Excellent Talents in University under Grant LR2015034.

Author Contributions: Taoyan Zhao conceived of and designed the experiments. Taoyan Zhao and Tian Li designed the control algorithm. Taoyan Zhao wrote the paper. Jiangtao Cao and Ping Li conducted the work.

Conflicts of Interest: The authors declare no conflict of interest.

\section{References}

1. Li, P.; Li, Q.A.; Lei, R.X. Development and application of advanced process control system for the ethylene cracking heaters. Chem. Ind. Eng. 2011, 62, 2216-2220.

2. Li, P.; Li, T.; Cao, J.T. Advanced Process Control of an Ethylene Cracking Furnace. Meas. Control 2015, 48, 50-53. [CrossRef] 
3. Kumbasar, T. A simple design method for interval type-2 fuzzy pid controllers. Soft. Comput. 2014, 18, 1293-1304. [CrossRef]

4. Kumbasar, T.; Eksin, I.; Guzelkaya, M.; Yesil, E. Interval type-2 fuzzy inverse controller design in nonlinear IMC structure. Eng. Appl. Artif. Intell. 2011, 24, 996-1005. [CrossRef]

5. Galluzzo, M.; Cosenza, B.; Matharu, A. Control of a Nonlinear Continous Bioreactor with Bifurcation by a Type-2 Fuzzy Logic Controller. Comput. Chem. Eng. 2008, 32, 2986-2993. [CrossRef]

6. Martinez, R.; Castillo, O.; Aguilar, L.T. Optimization of Interval Type-2 Fuzzy Logic Controllers for a Perturbed Autonomous Wheeled Mobile Robot Using Genetic Algorithms. Inf. Sci. 2009, 179, 2158-2174. [CrossRef]

7. Sanchez, M.A.; Castillo, O.; Castro, J.R. Generalized type-2 fuzzy systems for controlling a mobile robot and a performance comparison with interval type-2 and type-1 fuzzy systems. Expert Syst. Appl. 2015, 42, 5904-5914. [CrossRef]

8. Wu, D.; Tan, W.W. Genetic Learning and Performance Evaluation of Interval Type-2 Fuzzy Logic Controllers. Eng. Appl. Artif. Intell. 2006, 19, 829-841. [CrossRef]

9. Li, T.; Cao, J.T.; Li, P. Research of ethylene cracking furnace temperature control method based on IT2FIM. Chin. Comput. Appl. Chem. 2015, 32, 683-687.

10. Shahnazi, R. Observer-based adaptive interval type-2 fuzzy control of uncertain MIMO nonlinear systems with unknown asymmeteric saturation actuators. Inf. Sci. 2016, 171, 1053-1065.

11. Castillo, O.; Cervantes, L.; Soria, J.; Sanchez, M.; Castro, J.R. A generalized type-2 fuzzy granular approach with applications to aerospace. Inf. Sci. 2016, 354, 165-177. [CrossRef]

12. Sanchez, M.A.; Castillo, O.; Castro, J.R. Information granule formation via the concept of uncertainty-based information with interval type-2 fuzzy sets representation and Takagi-Sugeno-Kang consequents optimized with Cuckoo search. Appl. Soft Comput. 2015, 27, 602-609. [CrossRef]

13. Cervantes, L.; Castillo, O. Type-2 fuzzy logic aggregation of multiple fuzzy controllers for airplane flight control. Inf. Sci. 2015, 324, 247-256. [CrossRef]

14. Olivas, F.; Valdez, F.; Castillo, O.; Gonzalez, C.I.; Martinez, G.; Melin, P. Ant colony optimization with dynamic parameter adaptation based on interval type-2 fuzzy logic systems. Appl. Soft Comput. 2017, 53, 74-87. [CrossRef]

15. Tai, K.; EI-Sayed, A.R.; Biglarbegian, M.; Gonzalez, C.I.; Castillo, O.; Mahmud, S. Review of recent type-2 fuzzy controller applications. Algorithms 2016, 9, 39. [CrossRef]

16. Caraveo, C.; Valdez, F.; Castillo, O. Optimization of fuzzy controller design using a new bee colony algorithm with fuzzy dynamic parameter adaptation. Appl. Soft Comput. 2016, 43, 131-142. [CrossRef]

17. Kumbasar, T.; Eksin, I.; Guzelkaya, M.; Yesil, E. Type-2 Fuzzy Model Based Controller Design for Neutralization Processes. ISA Trans. 2012, 51, 277-287. [CrossRef] [PubMed]

18. Walker, C.L.; Walker, E.A. Sets with type-2 operations. Int. J. Approx. Reason. 2009, 50, 63-71. [CrossRef]

19. Molaeezadeh, S.F.; Moradi, M.H. A 2uFunction representation for non-uniform type-2 fuzzy sets: Theory and Design. Int. J. Approx. Reason. 2013, 54, 273-289. [CrossRef]

20. Castillo, O.; Amador-Angulo, L.; Castro, J.R.; Garcia-Valdez, M. A comparative study of type-1 fuzzy logic systems, interval type-2 fuzzy logic systems and generalized type-2 fuzzy logic systems in control problems. Inf. Sci. 2016, 354, 257-274. [CrossRef]

21. Coupland, S.; John, R. New geometric inference techniques for type-2 fuzzy sets. Int. J. Approx. Reason. 2008, 49, 198-211. [CrossRef]

22. Karnik, N.N.; Mendel, J.M. Centroid of a type-2 fuzzy set. Inf. Sci. 2001, 132, 195-220. [CrossRef]

23. Wu, H.; Mendel, J.M. Uncertainty bounds and their use in the design of interval type-2 fuzzy logic systems. IEEE Trans. Fuzzy Syst. 2002, 10, 622-639.

24. Garcia, C.E.; Morari, M. Internal model control. A unifying review and some new result. Ind. Eng. Chem. Process. Des. Dev. 1982, 21, 308-323. [CrossRef]

25. Liu, D.; Fujii, F. An adaptive interval model control system of a piezo-ceramic actuator with two RBF neural networks. In Proceedings of the 2014 IEEE Interval Conference on Mechatronics and Automation, Tianjin, China, 3-6 August 2014; pp. 210-215.

26. Toshiki, H.; Hiromitsu, O.; Hiroki, S.; Ryo, T.; Yoshihisa, I. Simplified internal model control for time delay processed. In Proceedings of the 35th Chinese Control Conference, Chengdu, China, 27-29 July 2016; pp. 308-312. 
27. Talange, D.B.; Laware, A.R.; Bandal, V.S. Development of an internal model sliding mode controller for cascade control system. In Proceedings of the 2015 International Conference on Energy Systems and Applications, Pune, India, 30 October-1 November 2015; pp. 51-56.

28. Sun, Z.P.; Chen, J.; Zhu, X.T. Multi-model internal model control applied in temperature reduction system. In Proceedings of the 11th World Congress on Intelligent Control and Automation, Shenyang, China, 29 June-4 July 2014; pp. 247-250.

29. Kumbasar, T.; Eksin, I.; Guzelkaya, M.; Yesil, E. Adaptive fuzzy internal model control design with bias term compensator. In Proceedings of the IEEE International Conference on Mechatronics, Kuala Lumpur, Malaysia, 17-19 May 2011; pp. 312-317.

30. Li, S.; Gu, H. Fuzzy adaptive internal model control schemes for PMSM speed-regulation system. IEEE Trans. Ind. Inform. 2012, 8, 767-779. [CrossRef]

31. Boukezzoula, R.; Galichet, S.; Folloy, L. Nonlinear internal model control: Application of inverse model based fuzzy control. IEEE Trans. Fuzzy Syst. 2003, 11, 814-829. [CrossRef]

32. Kumbasar, T.; Eksin, I.; Guzelkaya, M.; Yesil, E. Exact inversion of decomposable interval type-2 fuzzy logic systems. Int. J. Approx. Reason. 2013, 54, 253-272. [CrossRef]

33. Kumbasar, T.; Eksin, I.; Guzelkaya, M.; Yesil, E. An inverse controller design method for interval type-2 fuzzy models. Soft Comput. 2017, 21, 2665-2686. [CrossRef]

34. Kumbasar, T.; Eksin, I.; Guzelkaya, M.; Yesil, E. Type-2 fuzzy model inverse controller design based on BB-BC optimization method. In Proceedings of the 18th IFAC World Congress, Milano, Italy, 28 August2 September 2011; pp. 5308-5313.

35. Zadeh, L.A. Fuzzy Logic. Computer 1988, 21, 83-93. [CrossRef]

36. Karnik, N.N.; Mendel, J.M. Introduction to type-2 fuzzy logic systems. In Proceedings of the IEEE World Congress on IEEE International Conference on Fuzzy Systems, Anchorage, AK, USA, 4-9 May 1998; pp. 915-920.

37. Babuska, R. Fuzzy Modeling for Control; Springer Science Business Media, LLC.: New York, NY, USA, 1998; pp. 1593-1606.

38. Zhang, X.Y.; She, H.; Zheng, Y.Y. Operation optimization based on cyber-physical system for smart converter steelmaking. In Proceedings of the 35th Chinese Control Conference, Chengdu, China, 27-29 July 2016; pp. 6962-6966.

(C) 2017 by the authors. Licensee MDPI, Basel, Switzerland. This article is an open access article distributed under the terms and conditions of the Creative Commons Attribution (CC BY) license (http:/ / creativecommons.org/licenses/by/4.0/). 